\title{
Thermal stability of Fe/Mo layers
}

\author{
A. Liebig ${ }^{\mathrm{a}, *}$, B. Hjörvarsson ${ }^{\mathrm{a}}$, U. Rüdiger ${ }^{\mathrm{b}}$ \\ a Department of Physics, Uppsala University, Box 530, S-75121 Uppsala, Sweden \\ ${ }^{\mathrm{b}}$ Fachbereich Physik, Universität Konstanz, D-78457 Konstanz, Germany
}

Received 16 February 2005; received in revised form 2 August 2005; accepted 17 August 2005

Available online 28 September 2005

\begin{abstract}
The thermal stability of an iron/molybdenum bilayer was determined using Rutherford backscattering spectrometry and X-ray diffraction. Substantial interdiffusion (3\%) was observed upon annealing at $973 \mathrm{~K}$ for one hour. High temperature annealing of Fe/Mo layers in oxygen ambient is therefore not suitable for the formation of magnetite layers.
\end{abstract}

\section{Introduction}

The half-metallic $\mathrm{Fe}_{3} \mathrm{O}_{4}$ (magnetite) has attracted considerable interest, as it exhibits many of the properties required for successful realization of spintronic devices. Substantial work has therefore been invested on the growth of magnetite, with the goal to achieve maximum spin polarisation [1-5]. The growth has been performed by different techniques, for example reactive sputtering, plasma-assisted molecular beam epitaxy and oxidation of iron (110) surfaces.

High quality magnetite films can be obtained by high temperature oxidation of iron films $[2,6]$. This route is possible as the magnetite layers are almost perfectly lattice matched to the $\mathrm{Fe}$ layers and can therefore grow coherently without substantial defect generation in the oxide. Typically, a comparatively thick iron layer is grown and subsequently oxidised at temperatures around $970 \mathrm{~K}$ by oxygen exposures between 600 and 9000 Langmuir. The substrates used for the iron growth need therefore to fulfill two conditions: They have to have suitable lattice parameter for epitaxial growth of $\mathrm{Fe}$, and have to be chemically stable, hindering interdiffusion during the oxidation step. For single monolayers, where the exposure to high temperatures is short, $\mathrm{Pt}(111)$ [7] is suitable,

\footnotetext{
* Corresponding author. Fax: +46 184713524.

E-mail address: andreas.liebig@fysik.uu.se (A. Liebig).
}

for thicker films and longer oxidation steps $\operatorname{Mo}(110)$ and $\mathrm{W}(110)$, either as single crystals [2] or as thin films on sapphire substrates $[3,6]$, have been used.

The magnetite films prepared on molybdenum surfaces have previously been shown to exhibit significantly lower spin polarization, as compared to those prepared on tungsten substrates [3]. This is somehow surprising as the lattice parameter of Mo is closer to that of Fe. The structural quality of the Fe films is therefore expected to be better on Mo resulting in better quality of the magnetite layer. The most probable explanation to the reduced polarization is therefore the presence of uncompensated impurities in the Fe layers, which would require large interdiffusion of $\mathrm{Mo}$ in the Fe during the annealing process. This is supported by the binary phase diagram [8] which shows a Mo solubility of $3 \%$ in the $\alpha$-Fe phase at $970 \mathrm{~K}$. As a comparison, the solubility of $\mathrm{W}$ in $\mathrm{Fe}$ is below one percent at $1150 \mathrm{~K}$, with extremely slow kinetics. Although the binary phase diagram supports the presence of Mo in $\mathrm{Fe}$, the kinetics of the intermixing is unknown. Firm conclusion requires therefore direct measurements of the chemical stability of the $\mathrm{Mo} / \mathrm{Fe}$ layers, which are presented on the following pages.

\section{Experiment}

The investigated films were grown by molecular beam epitaxy in an ultra high vacuum system, with a base pressure in the $3^{*} 10^{-8} \mathrm{~Pa}$ range. The substrates used were $\mathrm{Al}_{2} \mathrm{O}_{3}(11 \overline{2} 0)$ 
single crystals $\left(10 \times 10 \mathrm{~mm}^{2}\right)$, cleaned in acetone and subsequently annealed in ultra high vacuum to $1073 \mathrm{~K}$ prior to deposition. The growth followed the recipe given in Ref. [9]. For details of growth see also Refs. [10,11].

The $15 \mathrm{~nm}$ thick molybdenum seed layers were deposited at $1073 \mathrm{~K}$. After cooling to room temperature, the iron films (thickness $25 \mathrm{~nm}$ ) were deposited, with a deposition rate of around $0.7 \AA / \mathrm{s}$. After deposition, the samples were annealed to $773 \mathrm{~K}$ for one hour in order to improve the crystallinity. The surface quality was investigated after each step using Low Energy Electron Diffraction (LEED). Two reference samples were also grown, one annealed at $773 \mathrm{~K}$ as described above, and a second one without any heat treatment after deposition.

The surface composition of the Fe layers upon annealing was investigated using Auger Electron Spectroscopy (AES). The AES was performed on one sample by alternating measurement and annealing steps at $973 \mathrm{~K}$ for $1200 \mathrm{~s}$ in an attempt to follow the eventual Mo enrichment on the surface.

Rutherford backscattering spectrometry (RBS) was used to determine the film composition. The RBS (ex situ) was performed with single charged $\mathrm{He}^{+}$ions at a primary beam energy of $2.00 \mathrm{MeV}$, at a scattering angle of $160^{\circ}$. The measurements were performed at different tilt angles, to avoid accidental channeling. The Rump software package from CGS [12], utilizing the algorithm described by Doolittle [13], was used for the analysis of the RBS data.

$\mathrm{X}$-ray diffractometry (XRD) was used to determine the outof-plane lattice parameter as well as the coherence length of the layers. The X-ray analysis was performed in a Bragg-Brentano geometry, using a commercial Siemens D 5000 diffractometer with $\mathrm{Cu} \mathrm{K} \alpha$ radiation.

\section{Results and discussion}

Annealing to $773 \mathrm{~K}$ developed clearly the rectangular LEED pattern of the Fe(110) surface as shown in Fig. 1. No changes

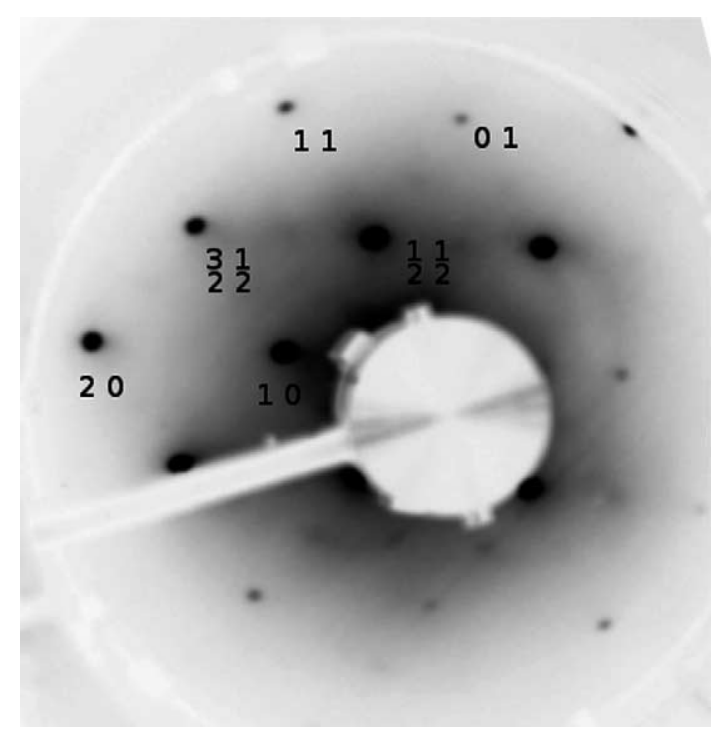

Fig. 1. LEED image of the Fe(110) surface. Sample slightly tilted with respect to the primary beam, electron energy $205 \mathrm{eV}$.

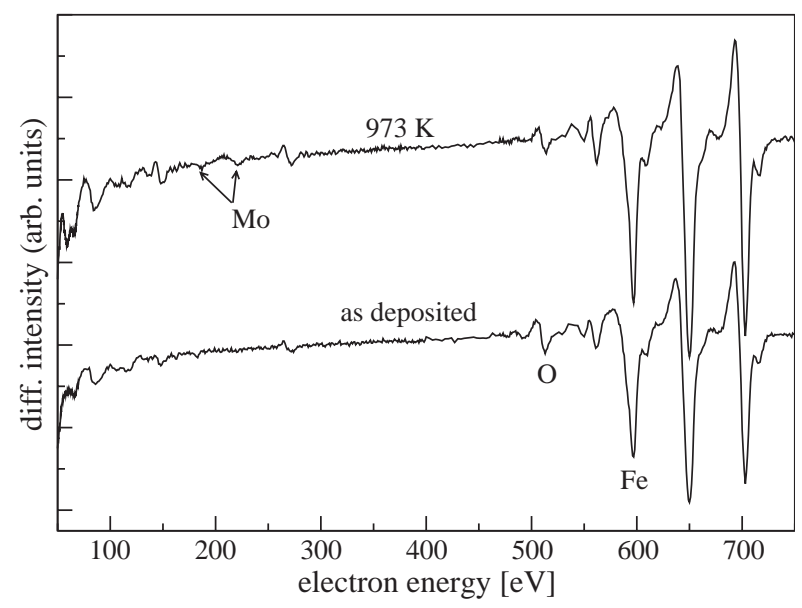

Fig. 2. Auger electron spectra, $3 \mathrm{keV}$ primary energy, normal incidence, before and after annealing.

were observed in the LEED pattern after the subsequent annealing steps. Since the LEED solely reflects the surface structure, XRD was used to investigate the crystalline quality of the films. The out-of-plane coherence length of the layers was determined from the full width at half maximum of the Mo and Fe peaks, using the Scherrer formula [14]. The resulting coherence lengths correspond to the thickness of the layers, hence, the quality is as good as it can be with respect to the out of plane coherency.

The change in the surface concentration of molybdenum upon annealing was determined using AES. No sign of Mo was observed after the initial annealing to $773 \mathrm{~K}$. After nine annealing steps to $973 \mathrm{~K}$, corresponding to total annealing time of $3 \mathrm{~h}$, the molybdenum MNN lines at $186 \mathrm{eV}$ and $221 \mathrm{eV}$ are still weak (see Fig. 2). Hence, only a low Mo concentration $(\approx 1$ at. $\%)$ is found at the Fe surface, even after long annealing times. However, even in case of equilibrium solution of Mo in $\mathrm{Fe}$, the surface concentration would be still close to the detection limit of this setup. Thus, a large Mo signal requires preferential surface segregation of Mo.

The degree of interdiffusion can conveniently be determined using RBS. Fig. 3 shows the results from a sample after

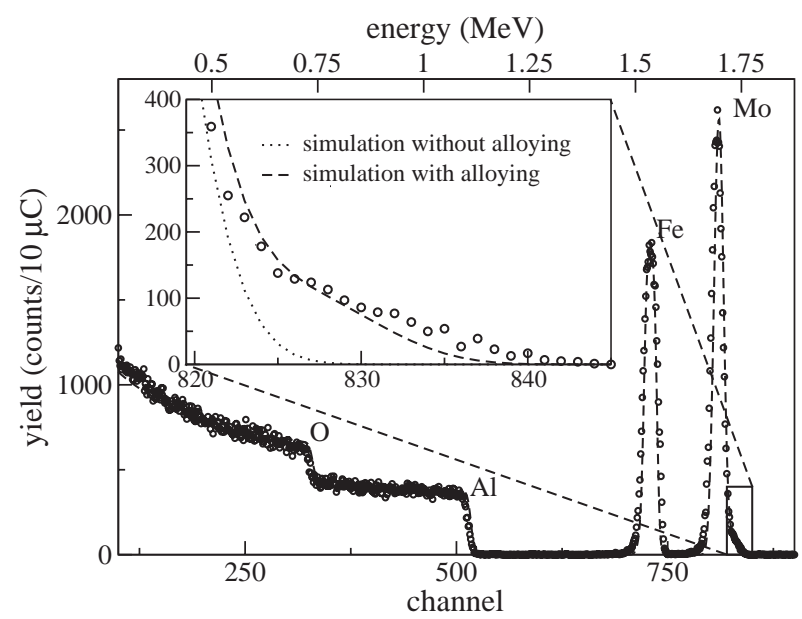

Fig. 3. RBS spectrum of annealed ( $973 \mathrm{~K}$ ) sample. Open circles show measured points. 


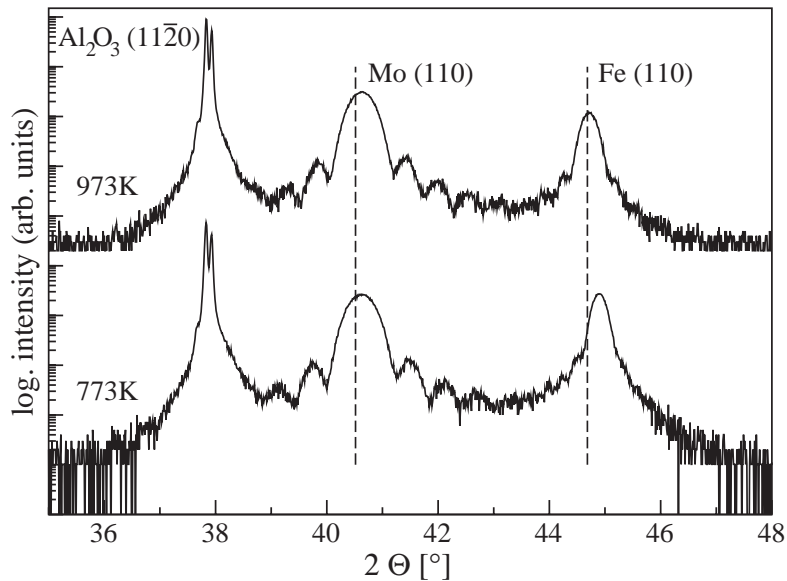

Fig. 4. X-ray diffraction patterns of samples annealed at different temperatures. The positions of the peaks for bulk Fe and Mo are marked.

annealing at $973 \mathrm{~K}$ for one hour. The signal from Mo and Fe, as well as the contribution from the substrate, is clearly separated. As seen in the figure, there is a significant shoulder at the Mohigh energy side (around 1.65 MeV), indicating the presence of Mo in the Fe layer. According to the simulations of the results, this contribution corresponds approximately to $3 \%$ of Mo in the Fe film. The results from the simulation are shown as an inset of the figure. Thickness variation of the Fe film, which could lead to similar RBS results, are not expected as the tailing is only seen in samples annealed at $973 \mathrm{~K}$. Furthermore, the XRD results are inconsistent with large a thickness variation as seen by the coherence length as well as the presence of the well defined Kiessig fringes.

Further proof comes from the changes in the average lattice parameters of the layers upon annealing. If Mo (bcc, $a=0.315$ $\mathrm{nm}$ ) is present in the Fe (bcc, $a=0.286 \mathrm{~nm}$ ) layers, it must influence the average lattice parameter of the Fe layer. With 3 at.\% of Mo in the Fe layers, the shift should be measurable using standard XRD equipment as long as Vegard's law is fulfilled. On the other hand, if the tailing of the Mo towards higher energies in the RBS spectrum would be due to thickness variations of the Fe layer, the $\mathrm{Fe}(110)$ atomic plane distance should be unaffected.

Fig. 4 shows the diffraction pattern of two of the investigated samples. The dashed lines mark the peak positions for bulk Fe(110) and $\mathrm{Mo}(110)$. A substantial shift of the iron (110) peak is observed between the two samples. Annealing to $973 \mathrm{~K}$ shifts the diffraction peak to lower angles, $d$-value of $2.025 \AA$, corresponding to a lattice expansion of $0.3 \%$.
Applying Vegard's law, the shift yields an average alloying of $3 \%$ to $4 \%$ of molybdenum into the iron, which is completely in line with the RBS results and the solubility of Mo in Fe. Thus, we conclude that the annealing to $973 \mathrm{~K}$ results in complete formation of the alpha phase of Mo in Fe.

\section{Conclusions}

The results unambiguously prove the diffusion of $\mathrm{Mo}$ in $\mathrm{Fe}$ films during annealing steps, required for the formation of good crystalline magnetite films. As the presence of Mo significantly reduces the spin polarization [3] the results disqualify high temperature annealing $(>970 \mathrm{~K})$ of $\mathrm{Mo} / \mathrm{Fe}$ layers as a step in the formation of highly spin-polarized magnetite films.

\section{Acknowledgements}

The Authors would like to thank C. Chacon for the help with the RBS measurements.

A. Liebig was supported during this work by a DAAD scholarship.

\section{References}

[1] W. Weiss, A. Barbieri, M.A. Van Hove, G.A. Somorjai, Phys. Rev. Lett. 71 (12) (1993) 1848.

[2] H.-J. Kim, J.-H. Park, E. Vescovo, Phys. Rev., B 61 (2000) 15284.

[3] M. Fonin, Y.S. Dedkov, J. Mayer, U. Rüdiger, G. Güntherodt, Phys. Rev., B 68 (2003) 045414.

[4] J.F. Bobo, D. Basso, E. Snoeck, C. Gatel, D. Hrabovsky, J.L. Gauffier, L. Ressier, R. Mamy, S. Visnovsky, J. Hamrle, J. Teillet, A.R. Fert, Eur. Phys. J., B Cond. Matter Phys. 24 (2001) 43.

[5] W. Kim, K. Kawaguchi, N. Koshizaki, M. Sohma, T. Matsumoto, J. Appl. Phys. 93 (10) (2003) 8032.

[6] Y. Dedkov, U. Rüdiger, G. Güntherodt, Phys. Rev., B 65 (2002) 064417.

[7] W. Weiss, G.A. Somorjai, J. Vac. Sci. Technol., A, Vac. Surf. Films 11 (4) (1993) 2138.

[8] Binary Alloy Phase Diagrams, second edition, ASM International, 1996.

[9] U. May, R. Calarco, J.O. Hauch, H. Kittur, M. Fonine, U. Rüdiger, G. Güntherodt, Surf. Sci. 489 (2001) 144.

[10] M. Tikhov, E. Bauer, Surf. Sci. 232 (1990) 73.

[11] J. Malzbender, M. Przybylsky, J. Giergel, J. Kirschner, Surf. Sci. 414 (1998) 187.

[12] M. Thompson, Rump-rbs analysis and simulation package (v 4.00(beta)), http://www.genplot.com, 2004.

[13] L.R. Doolittle, Nucl. Instrum. Methods Phys. Res., B Beam Interact. Mater. Atoms 15 (1985) 227.

[14] B.D. Cullity, Elements of X-Ray Diffraction, 2nd edition, AddisonWesley Publishing Company, Inc., 1978, p. 102, Ch. 3. 